\title{
Growth of non-modal transient structures during the spreading of surfactant coated films
}

\author{
Omar K. Matar and Sandra M. Troian ${ }^{\text {a) }}$ \\ Department of Chemical Engineering, Princeton University, Princeton, New Jersey 08544
}

(Received 21 January 1998; accepted 29 January 1998)

The spreading of surfactant coated thin liquid films is often accompanied by an instability producing significant film corrugation, fingering and branching. Marangoni stresses, responsible for the rapid and spontaneous spreading, are suspected as the main cause of unstable flow. Traditional eigenvalue analysis of a self-similar solution describing Marangoni driven spreading has predicted only stable modes. We present results of a transient growth study which reveals enormous amplification of initially infinitesimal disturbances in the film thickness. This analysis provides, for the first time, evidence of an instability resembling experimental patterns. (c) 1998 American Institute of Physics. [S1070-6631(98)02105-9]

Experiments by several independent groups ${ }^{1-4}$ (using both soluble and insoluble surfactants spreading on liquid films in different geometries) have confirmed the existence of a novel instability which produces inhomogeneous surface coverage. Gradients in surfactant concentration at the airliquid interface generate Marangoni stresses which produce rapid and spontaneous spreading. These stresses thin the liquid layer near the deposition region and draw surfactant and fluid into a thickened rim which advances rapidly over the uncontaminated film. Branched rivulets suddenly appear in the wake of the advancing rim as shown in Fig. 1.

A lubrication model has been developed to describe the spontaneous spreading of a surfactant coated drop ${ }^{5}$ or monolayer $^{6-9}$ along the surface of an uncontaminated thin liquid film. Analytic and numerical results for the film thickness successfully predict a rapidly advancing rim with subsequent thinning upstream and a monotonic decrease in surfactant concentration from the source to the leading edge. An earlier stability analysis which allowed only concentration disturbances and neglected variations in film thickness predicted unstable growth. ${ }^{5}$ A more recent calculation allowing self-consistent variations in both variables, however, has predicted asymptotic stability against disturbances of any wavenumber. ${ }^{10,11}$ These previous calculations examined the linear stability of self-similar solutions describing Marangoni driven spreading. It is now known, however, that the linearized operators governing the disturbance flow are highly non-normal. Their modal spectrum, therefore, can only determine the stability of the system as $t \rightarrow \infty$. A transient growth analysis is required to probe the flow characteristics at early times, as has been used to study many atmospheric and laboratory flows. ${ }^{12-14}$

Consider a quiescent Newtonian film of thickness $H_{0}$, viscosity $\mu$ and density $\rho$ resting on a solid horizontal substrate. The liquid is suddenly contacted by a monolayer of insoluble surfactant of initial extent $L_{0}$ where $\varepsilon=H_{0} / L_{0}$ $\ll 1$ in accordance with the lubrication approximation. The spontaneous spreading reflects the balance between the surface shear stress of order $\Pi / L_{0}$ and the viscous drag of order $\mu U / H_{0}$. The parameter $\Pi=\sigma_{0}-\sigma_{m}$ defines the spreading coefficient where $\sigma_{0}$ is the surface tension of the clean liquid and $\sigma_{m}$ the initial surface tension of the coated liquid. The characteristic spreading velocity, $U$, is determined from the force balance to be $U=\varepsilon \Pi / \mu$. The equations of motion are non-dimensionalized by the horizontal scale, $L_{0}$, the vertical scale, $H_{0}$, the horizontal velocity, $U$ and the vertical velocity, $\varepsilon U$. The characteristic scales for time and pressure are $\mu L_{0} / \varepsilon \Pi$ and $\Pi / H_{0}$, respectively. The dimensionless surface tension is defined to be $\left(\sigma-\sigma_{0}\right) / \Pi$. For parameter values of relevance, gravitational and disjoining pressure effects are negligible. They can be incorporated in a straightforward fashion when relevant. Since streamwise spreading rates are very rapid, for computational efficiency we define a stretched coordinate, $\xi=x / L(\tau)$, where $x$ represents the horizontal coordinate, $\tau$ represents a dimensionless time, and $L(\tau)$ locates the extent of the surfactant coated film. We consider the simplest case of a finite surfactant monolayer spreading in rectilinear geometry ${ }^{8,9}$ for which $L(\tau) \sim \tau^{1 / 3}$. The rescaled one-dimensional (1-D) base state equations for the film thickness, $h(\xi, \tau)$, and the surfactant concentration, $g(\xi, \tau) / \tau^{1 / 3}$, including Marangoni, surface diffusion and capillary forces are given by ${ }^{8,10,11}$

$$
\begin{aligned}
\tau h_{\tau}= & \frac{1}{3} \xi h_{\xi}+\frac{1}{2}\left(h^{2} g_{\xi}\right)_{\xi}-\left(\mathscr{C} / 3 \tau^{1 / 3}\right)\left(h^{3} h_{\xi \xi \xi}\right)_{\xi}, \\
\tau g_{\tau}= & \frac{1}{3}(\xi g)_{\xi}+\left(g h g_{\xi}\right)_{\xi}+\left(\tau^{1 / 3} / P e_{s}\right) g_{\xi \xi}-\left(\mathscr{C} / 2 \tau^{1 / 3}\right) \\
& \times\left(g h^{2} h_{\xi \xi \xi \xi}\right)_{\xi},
\end{aligned}
$$

where subscripts represent partial differentiation. The effective capillary number is defined by $\mathscr{C}=\varepsilon^{2} \sigma_{m} / \Pi$ while the effective Peclet number is $P e_{s}=\Pi H_{0} / \mu \mathscr{D}_{s}$, where $\mathscr{D}_{s}$ represents the surfactant diffusion coefficient along the interface. Although the dimensionless group $\mathscr{C}$ scales with $\varepsilon^{2}$, it cannot be neglected within the lubrication approximation ${ }^{15}$ since it multiplies terms which achieve magnitudes of order $\varepsilon^{-2}$.

Linearizing about the solutions of Eqs. (1) and (2) to include 2-D disturbances of the form $(\widetilde{h}, \widetilde{g})=(\psi, \phi)$ 


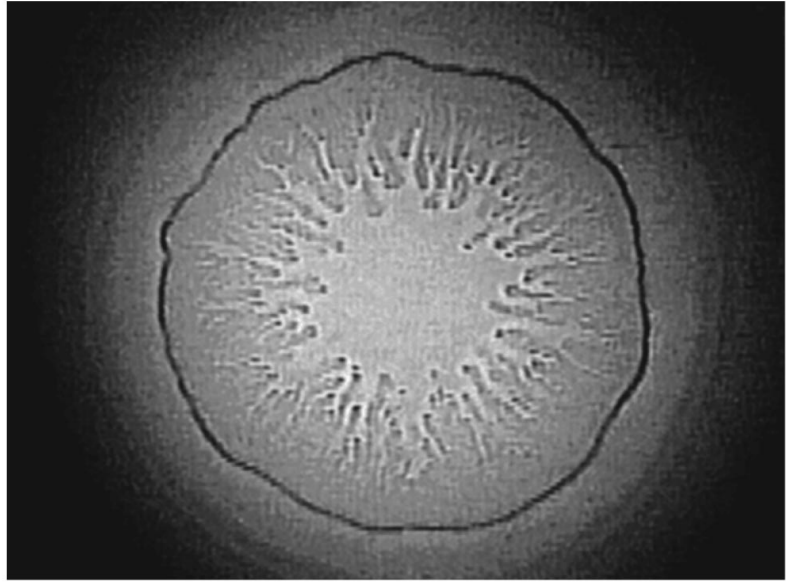

FIG. 1. Pattern observed during the spreading of $6.6 \mathrm{mM}$ aqueous anionic SDS solution on water film $3 \mathrm{sec}$ after deposition (volume $=15 \mu \mathrm{l}, H_{0}$ $\approx 1 \mu$, concentration $=6.6 \mathrm{mM}$. Dark outer ring of diameter $9.6 \mathrm{~cm}$ demarcates leading edge of spreading film. Similar patterns occur with other soluble or insoluble surfactants spreading on water or other viscous liquids.

$\times(\xi, \tau) \cos (K z)$, where $K$ denotes the disturbance wavenumber and $z$ the unstretched transverse coordinate, yields the two coupled equations:

$$
\begin{aligned}
\tau \psi_{\tau}= & \mathscr{B}_{1}[\psi, \phi] \\
= & \frac{1}{3} \xi \psi_{\xi}+\frac{1}{2}\left(h^{2} \phi_{\xi}+2 h g_{\xi} \psi\right)_{\xi}-\left[\left(K \tau^{1 / 3}\right)^{2} / 2\right] h^{2} \phi \\
& -\left(\mathscr{C} / 3 \tau^{1 / 3}\right)\left[\left(h^{3} \psi_{\xi \xi \xi}+3 h^{2} h_{\xi \xi \xi} \psi\right)_{\xi}\right]+\left(\mathscr{C} / 3 \tau^{1 / 3}\right) \\
& \times\left[\left(K \tau^{1 / 3}\right)^{2}\left(\left(h^{3}\right)_{\xi} \psi_{\xi}+2 h^{3} \psi_{\xi \xi}\right)-\left(K \tau^{1 / 3}\right)^{4} h^{3} \psi\right],
\end{aligned}
$$

$$
\begin{aligned}
\tau \phi_{\tau}= & \mathscr{L}_{2}[\psi, \phi] \\
= & \frac{1}{3}(\xi \phi)_{\xi}+\left(g g_{\xi} \psi+h g_{\xi} \phi+h g \phi_{\xi}\right)_{\xi}-\left(K \tau^{1 / 3}\right)^{2} h g \phi \\
& +\left(\tau^{1 / 3} / P e_{s}\right)\left(\phi_{\xi \xi}-\left(K \tau^{1 / 3}\right)^{2} \phi\right)-\left(\mathscr{C} / 2 \tau^{1 / 3}\right) \\
& \times\left[\left(g h^{2} \psi_{\xi \xi \xi}+2 g h h_{\xi \xi \xi} \psi+h^{2} h_{\xi \xi \xi} \phi\right)_{\xi}\right] \\
& +\left(\mathscr{C} / 2 \tau^{1 / 3}\right)\left[\left(K \tau^{1 / 3}\right)^{2}\left(\left(g h^{2}\right)_{\xi} \psi_{\xi}+2 g h^{2} \psi_{\xi \xi}\right)\right. \\
& \left.-\left(K \tau^{1 / 3}\right)^{4} g h^{2} \psi\right] .
\end{aligned}
$$

Explicit computation of the commutator for $\mathscr{L}_{1}$ and $\mathscr{L}_{2}$ reveals that these disturbance operators are highly non-normal. The method of lines, which consists of a second order difference scheme with Gear's method for time integration, was used to solve the system of Eqs. (1)-(4) for various parameter values of $P e_{s}, \mathscr{C}$ and $K$. The number of grid points ranged from 201 to 301; convergence was achieved upon mesh refinement. For the results presented herein, the initial base state profiles starting at time $\tau=1$ correspond to a flat film, $h(\xi, 1)=1$, and a smoothly decaying surfactant concentration, $g(\xi, 1)=0.5[1-\tanh (10(\xi-0.5))]$. The initial disturbance profiles are Gaussian shaped in the streamwise direction and centered ahead of the deposited film at $\xi=1.0$ with form $\psi(\xi, 1)=\phi(\xi, 1)=e^{-10(\xi-1.0)^{2}}$. Since the disturbance equations are linear, the amplitude of $\psi$ and $\phi$ can be

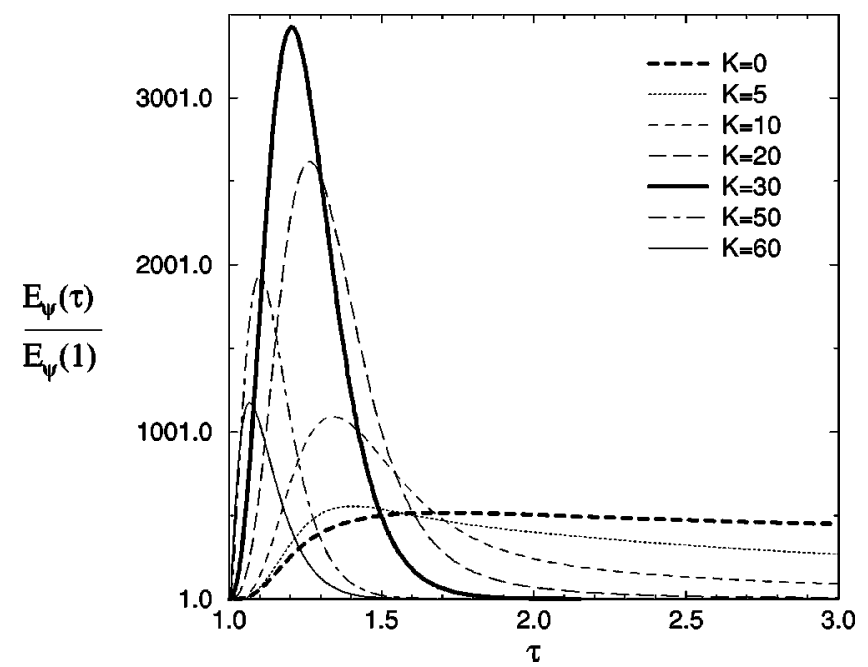

FIG. 2. The disturbance amplification for parameter values $P e_{s}=5000$ and $\mathscr{C}=10^{-5}$ for wavenumbers in the range $0 \leqslant K \leqslant 60$.

set to unity. We are presently investigating other forms of initial conditions ${ }^{16}$ but the features described below characterize the typical response of the system.

To quantify the degree of deformation during the spreading process, we examine the $\operatorname{ratio}^{17} E_{\Psi}(\tau)$ $\equiv \int_{0}^{\infty} \psi^{2}(\xi, \tau) d \xi / \int_{0}^{\infty} h^{2}(\xi, \tau) d \xi$ which represents a generalized mechanical energy. Figure 2 shows the development and decay of this quantity for $P e_{s}=5000$ and $\mathscr{C}=10^{-5}$, parameter values consistent with experiments ${ }^{1}$. The $K=30$ mode achieves the largest amplification while the $K=60$ mode achieves the largest growth rate. The amplification shown increases in value when the disturbance is placed further ahead of the initial surfactant distribution. In this case the film thickness has a longer time to evolve and develops a steeper front before encountering the perturbation. This extreme sensitivity to small disturbances occurs despite the fact that the mobility contrast due to variations in the film thickness is not large; the rim is at most twice as high as the original undisturbed flat film. This feature is in stark contrast to the stability of a thin viscous film flowing down an inclined plane wherein transient amplification all but disappears when the leading edge is twice as high as the undisturbed precursor film ${ }^{18}$ (see Fig. 11 in Ref. 13). In summary, parameter values which increase the initial shear stress, which increase the spreading speed or which steepen the shape of the advancing front (i.e., by increasing $P e_{s}$ or decreasing $\mathscr{C}$ ) produce correspondingly larger amplification ratios. In all cases, the $K=0$ mode maintains the largest amplification ratio for long times eventually decaying to zero as $\tau \rightarrow \infty$, in agreement with our previous modal analysis ${ }^{10,11}$ which predicts asymptotic stability.

To isolate the mechanism leading to instability we have also examined the rate of energy production. ${ }^{16}$ The mechanism which produces significant corrugation in the film thickness with subsequent striations can be traced to the relative strength of the Marangoni stresses compared to weaker surface diffusion and capillary forces. Consider a transverse sinusoidal perturbation in the film thickness and surfactant concentration. Regions at the crest of the original distur- 
(a)

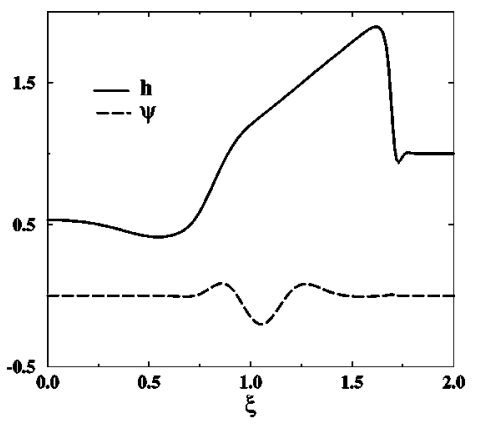

(b)

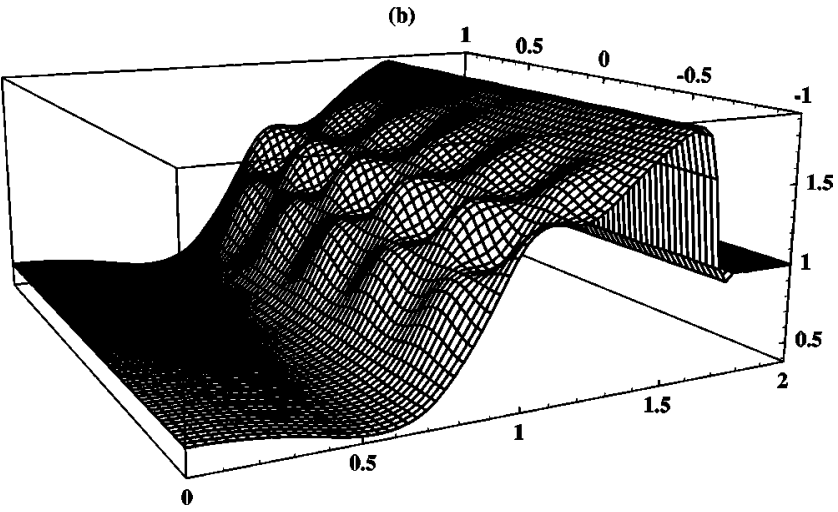

FIG. 3. (a) An example of the base state profile, $h(\xi, \tau)$, and associated disturbance, $\psi(\xi, \tau)$, of wavenumber $K=5$ for $P e_{s}=5000$ and $C=10^{-5}$ at $\tau=7$. (b) 3-D visualization of total film thickness for the same parameter values.

bance, which represent the thickest portions of the film and those laden with most surfactant, are severely thinned by the rapid Marangoni flow of surfactant and fluid toward the downstream and transverse directions. The troughs are fed by the transverse flow and thicken to produce elevated streaks in the streamwise direction. Capillarity and surface diffusion oppose both trends but are insufficient to reestablish equilibrium on a transient time scale. ${ }^{19}$ In the frame of reference of the advancing rim, the disturbances first migrate toward the rim but then lag progressively behind. Figure $3 \mathrm{~b}$ shows an example of the total film thickness at $\tau=7.0$ (chosen to correspond to the time after deposition shown in Fig. 1) for an applied disturbance of $K=5$. The transient disturbance produces slender interlocking streamlets which have localized behind the advancing front. As indicated in Fig. 2, the disturbances eventually decay in amplitude as the magnitude of the Marangoni stresses decreases with time due to dilution, which is consistent with experimental observations.

Although perturbations of all wavenumbers eventually decay, the explosive transient growth may trigger a nonlinear response leading to unstable flow and finger formation. We have initiated direct simulations of the non-linear 2-D evolution equations to address issues related to mode coupling and film branching with tip-splitting, features which cannot be captured within a linearized model.

\section{ACKNOWLEDGMENTS}

S.M.T. gratefully acknowledges support from the National Science Foundation (RIA and CAREER programs), a seed grant from the Princeton Materials Institute, the Exxon Education Foundation and the Can Manufacturer's Institute.

${ }^{a)}$ Correspondence should be e-mailed to S.M.T. at stroian@princeton.edu

${ }^{1}$ S. M. Troian, X. L. Wu, and S. A. Safran, "Fingering instability in thin wetting films,' Phys. Rev. Lett. 62, 1496 (1989).

${ }^{2}$ S. Zhu, W. G. Miller, L. E. Scriven, and H. T. Davis, "Superspreading of water-silicone surfactant on hydrophobic surfaces,' Colloids Surf., A 90, 63 (1994).

${ }^{3} \mathrm{~S}$. He and J. Ketterson, "Surfactant driven spreading of a liquid on a vertical surface,', Phys. Fluids A 7, 2640 (1995).

${ }^{4}$ B. Frank and S. Garoff, "Origins of the complex motion of advancing surfactant solutions,', Langmuir 11, 87 (1995).

${ }^{5}$ S. M. Troian, E. Herbohlzheimer, and S. A. Safran, "Model for the fingering instability of spreading surfactant drops,', Phys. Rev. Lett. 65, 333 (1990).

${ }^{6}$ M. E. Borgas and J. B. Grotberg, "Monolayer flow on a thin film," J. Fluid Mech. 193, 151 (1988).

${ }^{7}$ D. P. Gaver and J. B. Grotberg, "The dynamics of a localized surfactant on a thin film,' J. Fluid Mech. 213, 127 (1990).

${ }^{8}$ O. E. Jensen and J. B. Grotberg, "Insoluble surfactant spreading on a thin viscous film: Shock evolution and rupture,' J. Fluid Mech. 240, 259 (1992).

${ }^{9}$ F. F. Espinosa, A. H. Shapiro, J. J. Fredberg, and R. D. Kamm, "'Spreading of exogenous surfactant in an airway,' J. Appl. Physiol. 75, 2028 (1993).

${ }^{10}$ O. K. Matar and S. M. Troian, in Dynamics in Small Confining Systems III, Material Research Society Symposium Proceedings, edited by J. M. Drake, J. Klafter, and E. R. Kopelman (Materials Research Society, Boston, 1996), Vol. 464, p. 237.

${ }^{11}$ O. K. Matar and S. M. Troian, "Linear stability analysis of an insoluble surfactant monolayer spreading on a thin liquid film,' Phys. Fluids A 9, 3645 (1997).

${ }^{12}$ B. F. Farrell and P. J. Ioannou, "Generalized stability theory. Part II: Nonautonomous operators,', J. Atmos. Sci. 53, 2041 (1996), and references therein.

${ }^{13}$ A. L. Bertozzi and M. P. Brenner, "'Linear stability analysis and transient growth in driven contact lines,'” Phys. Fluids A 9, 530 (1997).

${ }^{14}$ Recent work by Ye and Chang ${ }^{20}$ and Johnson et al., ${ }^{21}$ however, fails to confirm the hypothesis that transient growth of microscopic disturbances at the contact line produces the rivulets observed in flows down an inclined plane.

${ }^{15}$ D. R. Wilson and A. F. Jones, "The entry of a falling film into a pool and the air-entrainment problem,'’ J. Fluid Mech. 128, 219 (1983).

${ }^{16}$ O. K. Matar and S. M. Troian, "Transient growth analysis of the spreading of surfactant coated liquid films," to be submitted to Phys. Fluids.

${ }^{17}$ S. F. Shen, "Some considerations of the laminar stability of incompressible time-dependent basic flow,', J. Aerosp. Sci. 28, 397 (1961).

${ }^{18}$ In addition, the mechanism for instability is markedly different and produces different flow patterns. Whereas the surfactant fingering patterns are highly branched, dendritic-like structures, the falling film produces simple straight-edged rivulets which never bifurcate.

${ }^{19} \mathrm{We}$ have also found that while gravitational forces exert an additional stabilizing influence, van der Waals forces strongly enhance transient disturbances.

${ }^{20}$ Y. Ye and H.-C. Chang, "Fingering instability on a prewetted plane," Division of Fluid Dynamics Meeting, American Physical Society, Abstract Dd.03, 1997.

${ }^{21}$ M. Johnson, R. Schluter, M. Miksis, and S. Bankoff, "'Experimental study of rivulets,' Division of Fluid Dynamics Meeting, American Physical Society, Abstract Dd.02, 1997. 